\title{
Ambiguities in the Tsallis description of non-thermal plasma species
}

\author{
FRANK VERHEEST \\ Sterrenkundig Observatorium, Universiteit Gent, Krijgslaan 281, B-9000 Gent, Belgium \\ School of Chemistry and Physics, University of KwaZulu-Natal, Durban 4000, South Africa \\ (frank.verheest@ugent.be)
}

(Received 22 August 2013; accepted 2 September 2013; first published online 21 October 2013)

\begin{abstract}
Tsallis $q$-non-extensive distributions have recently found favor in describing the presence of energetic particles and their influences on several plasma modes, notably electrostatic solitons. Here attention is drawn to ambiguities and subtleties in the superextensive and subextensive ranges in $q$, which are not always recognized in the literature, particularly in numerical studies.
\end{abstract}

\section{Introduction}

The presence of energetic particles in plasmas, resulting in long-tailed distributions, is an intrinsic element in many space and laboratory observations. Different models have been proposed to describe this effect, which do not address the actual mechanisms responsible for particle acceleration but rather model their effects on wave dynamics via phenomenological modification of electron or other hot species distribution functions.

Among the earlier deviations from pure Maxwellians were the kappa and Cairns non-thermal distributions. The kappa distributions (Vasyliunas 1968; Hellberg et al. 2009) were introduced by Vasyliunas (1968) to fit phenomenologically the power law-like dependence of electron distributions observed in space. Cairns et al. (1995) introduced a distribution designed to model enhanced high-energy tails that are frequently observed in space plasmas. It showed that the nature of ion sound solitary structures can change in the presence of non-thermal electrons, producing nonlinear solitary waves that may have either enhanced or depleted density.

More recent was the introduction of $q$-non-extensive Tsallis distributions (Tsallis 1988) in the description of nonlinear plasma waves (see, e.g. Lima et al. 2000). Interestingly, it has been argued that the kappa scenario derives from the Tsallis distribution (Livadiotis and McComas 2009; Livadiotis and McComas 2011), although this speculative analogy is rather phenomenological and remains the subject of much debate (Hellberg et al. 2009; Livadiotis and McComas 2011). This shall not be pursued here as it is beyond the focus on specific properties of the Tsallis distributions.

Typical Tsallis non-extensive $q$-distributions for hot plasma species with non-thermal properties have the homogeneous equilibrium form (Lima et al. 2000)

$$
f(v)=C\left[1-\frac{1}{2}(q-1) v^{2}\right]^{1 /(q-1)},
$$

where $v$ is the non-dimensional phase space velocity in units of $T / m, q$ is the non-extensive index, and $C$ is a $q$-dependent normalization constant, to be determined below. The hot species under consideration have kinetic temperature $T$ (in the absence of non-extensive effects) and mass $m$. Such homogeneous equilibrium distributions contain neither time $(t)$ nor space $(x)$ coordinates. Moreover, we will discuss (1.1) for one-dimensional problems in order to keep the algebra to its simplest expression. Indeed, the three-dimensional versions suffer from similar ambiguities as explained here for the onedimensional variant, and applications in the plasma soliton theory almost automatically take an approach with only one space dimension.

Preliminary remarks are that for $q \rightarrow 1,(1.1)$ reduces to the well-known Maxwellian distribution in terms of $\exp \left(-v^{2} / 2\right)$. Furthermore, for $q<1$ the expression between the square brackets in (1.1) is strictly positive for all $v$, and hence can serve to model superthermal wings in the distribution function. However, for $q>1$ this is no longer the case, and (1.1) is only defined in the range $-v_{\max } \leqslant v \leqslant+v_{\max }$, with

$$
v_{\max }=\sqrt{\frac{2}{q-1}} .
$$

Thus, $f(v)$ has to be taken zero for $|v|>v_{\max }$, implying that (initially) there are no particles with velocities larger than $v_{\max }$, which renders the range $q>1$ unsuitable to model superthermality. We shall deal with the two $q$ ranges separately, leaving the limit $q=1$ (amounting to the well-known Maxwellian distribution) out of this discussion. The two ranges in $q$ also correspond to the distinction between superextensivity $(q<1)$ and subextensivity $(q>1)$ (Saberian and Esfandyari-Kalejahi 2013) in terms of the Tsallis entropy (Tsallis 1988), details of which are beyond the scope of the present paper. 


\section{Case $q<1$}

For $q<1$ there is no limitation on $v$, and $f(v)$ is defined for all $-\infty \leqslant v \leqslant+\infty$, as are Maxwellian, Cairns non-thermal, and kappa superthermal distributions, to quote some of the other distribution functions frequently encountered in the relevant literature.

In order to obtain a finite, physically realistic normalization, when integrating $f(v)$ over all $v$, one needs to satisfy the stringent requirement that $f(v) \rightarrow 0$ faster than $|v|^{-1}$ for $|v| \rightarrow \infty$. Since $f(v) \propto|v|^{2 /(q-1)}$ for $|v| \rightarrow \infty$, the integral converges, provided $-1<q$. Together this yields $-1<q<1$, often quoted in the literature without further ado.

Start with the determination of $C$, for the case $-1<$ $q<1$, by imposing that

$$
\int_{-\infty}^{+\infty} f(v) d v=1
$$

This choice ensures that the undisturbed hot species density is normalized to 1 , but another constant could be chosen as in plasmas with more than two species. However, this only changes the expression for $C$ by a constant multiplicative factor, and constitutes no essential restriction for the present discussion. Substituting (1.1) into (2.1) yields

$$
C_{A}=\sqrt{1-q} \Gamma\left(\frac{1}{1-q}\right) / \sqrt{2 \pi} \Gamma\left(\frac{q+1}{2(1-q)}\right)
$$

in terms of gamma functions $\Gamma$. The label $A$ serves to identify the range $-1<q<1$, and we note that for $q \rightarrow 1, C_{A}$ reduces to $1 / \sqrt{2 \pi}$.

Having established the homogeneous equilibrium distribution function and its limits, we next wish to compute the hot species density in the presence of an electrostatic field disturbance with normalized potential $\varphi(x, t)$. The standard technique is that one replaces $v^{2}$ by $v^{2}-2 \varphi(x, t)$ and integrates over the allowable range in $v$. The minus sign in $v^{2}-2 \varphi(x, t)$ is for Tsallis electrons, the most common case, and must be replaced by a plus sign for positive Tsallis ions, accompanied by adaptations of the normalization. If there is more than one Tsallis species, labels and parameters involving changes in the normalization have to be introduced, but these are technicalities and do not influence the fundamental remarks that follow. To lighten the notation somewhat, the dependence of $\varphi$ on $x$ and $t$ will not be written explicitly, but we need to keep in mind that this is a function in physical space and not simply a constant.

Proceeding with Tsallis electrons, the normalized electron density is obtained as

$$
\begin{aligned}
n_{A} & =\int_{-\infty}^{+\infty} C_{A}\left[1-\frac{1}{2}(q-1)\left(v^{2}-2 \varphi\right)\right]^{1 /(q-1)} \\
& =[1+(q-1) \varphi]^{(q+1) / 2(q-1)}
\end{aligned}
$$

The subscript $A$ reminds us that this is valid for $-1<$ $q<1$, and the density (2.3) is commonly found in the literature, but often without mention of the restrictions on $q$.

Another step in the integration process is the determination of the energy, involving the integral of $v^{2} f(v)$ over all $v$. For this to be finite, we apply a reasoning similar to that presented for the normalization: now $v^{2} f(v)$ has to go to zero faster than $|v|^{-1}$ for $|v| \rightarrow \infty$. Since $v^{2} f(v) \propto|v|^{2 q /(q-1)}$ for $|v| \rightarrow \infty$, the integral converges, provided $1 / 3<q$, and indeed

$$
E_{A}=\frac{1}{2} \int_{-\infty}^{+\infty}\left(C_{A} / C\right) v^{2} f(v) d v=\frac{1}{3 q-1} .
$$

This plausible requirement for having a finite energy means that the acceptable range for $q$ is necessarily narrowed to $1 / 3<q<1$, a result that was also arrived at in different contexts of Langmuir oscillations (Saberian and Esfandyari-Kalejahi 2013), but which is often not adhered to in numerical evaluations. This is in contrast to kappa distribution theory, where the argument that the energy should remain finite has led to the standard requirement that $\kappa>3 / 2$ (Vasyliunas 1968; Hellberg et al. 2009).

Further restrictions occur when higher, even moments are considered, since demanding that $v^{2 p} f(v) \rightarrow 0$ faster than $|v|^{-1}$ for $|v| \rightarrow \infty$ leads to $(2 p-1) /(2 p+1)<q$. Indeed, for the normalization $p=0$ gives $-1<q$, for the energy $p=1$ gives $1 / 3<q$ and for, e.g. $p=2$ one gets $3 / 5<q$, and so on. Since $f(v)$ is even in $v$, odd moments vanish after integration over all $v$, but keeping higher even moments finite severely restricts the range in $q$ below 1. To the best of our knowledge, this aspect has not been discussed in the literature, and surfaced only recently when a critical appraisal was given (Williams et al. 2013) of a newfangled combined Cairns-Tsallis distribution (Tribeche et al. 2012).

It should be remarked here that both the Maxwellian and the Cairns non-thermal distributions do not suffer from this problem, since the exponential $\exp \left(-v^{2} / 2\right)$ ensures that all moments are finite. The kappa distributions, on the other hand, may exhibit similar convergence effects in reducing the allowed kappa range for higher moments, although this aspect has not been investigated.

\section{Case $q>1$}

Now the second range for $q$, namely $q>1$ is addressed. Because the velocity range is restricted to a finite interval, the problem of how $f(v)$ behaves at very large $|v|$ does not arise when computing the normalization or other moments. At the same time, this finite basis means that the Tsallis distributions with $q>1$ cannot model plasmas with superthermal wings. From

$$
\int_{-v_{\max }}^{+v_{\max }} f(v) d v=1
$$


not surprisingly, a quite different form for $C$ is derived,

$$
C_{B}=(q-1)^{3 / 2} \Gamma\left(\frac{1}{2}+\frac{q}{q-1}\right) / \sqrt{2 \pi} \Gamma\left(\frac{1}{q-1}\right) .
$$

Expressions (2.2) and (3.2) correspond to what is found in the literature (see, e.g. Saberian and EsfandyariKalejahi 2013 and many others).

However, determining the appropriate hot species density for $q>1$ is more complicated, since one has to compute

$$
\int_{-v_{B}}^{+v_{B}} C_{B}\left[1-\frac{1}{2}(q-1)\left(v^{2}-2 \varphi\right)\right]^{1 /(q-1)},
$$

where the upper and lower integration limits, $\pm v_{B}$, need to be carefully discussed. The expression under the integration sign now includes the influence of electrostatic potential $\varphi$, which means that we are no longer dealing with the undisturbed equilibrium distribution. Hence, one cannot bluntly use $v_{B}=v_{\max }$ as given by (1.2) because for, e.g. positive $\varphi$, a density would be obtained, given by

$$
\begin{aligned}
& C_{B} \frac{2 \sqrt{2}}{\sqrt{q-1}}[1+(q-1) \varphi]^{1 /(q-1)} \\
& \quad \times{ }_{2} F_{1}\left[\frac{1}{2}, \frac{1}{1-q}, \frac{3}{2}, \frac{1}{1+(q-1) \varphi}\right],
\end{aligned}
$$

where ${ }_{2} F_{1}$ is the Gauss hypergeometric function. Rather, it is noted that the integrand in (3.3) is well defined in the range

$$
-\sqrt{\frac{2[1+(q-1) \varphi]}{q-1}} \leqslant v \leqslant+\sqrt{\frac{2[1+(q-1) \varphi]}{q-1}} .
$$

Hence, taking now $v_{B}=\sqrt{2[1+(q-1) \varphi] /(q-1)}$ yields

$$
\begin{aligned}
n_{B} & =\int_{-v_{B}}^{+v_{B}} C_{B}\left[1-\frac{1}{2}(q-1)\left(v^{2}-2 \varphi\right)\right]^{1 /(q-1)} \\
& =[1+(q-1) \varphi]^{(q+1) / 2(q-1)} .
\end{aligned}
$$

Rather surprisingly, this is precisely the same expression as a function of $\varphi$ as found for $n_{A}$ in (2.3)! The ambiguity underlying all this is that in the absence of any disturbance, the allowable velocity range in equilibrium is limited by constant $\pm v_{\max }$, and that the presence of electrostatic effects can be interpreted as expanding or shrinking this range, according to whether the electrons acquire or lose energy in the wave. However, one does not often deal with an undisturbed distribution whose definition range then changes according to possible disturbances, which are space- and time-dependent.

For the energy one finds that

$$
E_{B}=\frac{1}{2} \int_{-v_{\max }}^{+v_{\max }}\left(C_{B} / C\right) v^{2} f(v) d v=\frac{1}{3 q-1} .
$$

This is the same result as in (2.4), but now $q>1$, and thus $E_{B}$ is automatically positive, contrary to $E_{A}$, where the restriction $1 / 3<q$ was needed to ensure that.
Given the different ranges in $q$, it is easily seen that $E_{A}>1 / 2>E_{B}$, indicating that the superthermal wings can substantially increase the total energy in the case $1 / 3<q<1$.

Finally, we might add that in several papers the authors immediately start from a distribution which includes the potential energy,

$$
C\left[1-\frac{1}{2}(q-1)\left(v^{2}-2 \varphi\right)\right]^{1 /(q-1)} .
$$

While this is non-sensical for a homogeneous equilibrium, such distribution functions depend on $x, t, v$ through $\varphi(x, t)$. Then for $q>1$ one proceeds to integrate (3.8) in $v$ over the range indicated in (3.5) and fixes $C$ through the requirement that $n_{B}=1$ in equilibrium, at $\varphi=0$, which leads to $C=C_{B}$ as given in (3.2). However, for the even moments of $f(v)$ one has to take the integration limits as $\pm v_{\max }$, since $\varphi$ is not included in the computation.

\section{Conclusions}

To summarize, attention has been drawn to several ambiguous or subtle aspects of Tsallis distributions as used in the context of electrostatic soliton descriptions in plasma physics. For the superextensive range $-1<q<1$, requiring the energy to be finite means that the allowable range shrinks to $1 / 3<q<1$, and even further when higher velocity moments of the undisturbed distribution functions are needed. The subextensive range $q>1$ is afflicted by ambiguities, when hot species densities are computed, in that the velocity range $-v_{\max } \leqslant v \leqslant v_{\max }$, derived from considering the undisturbed distribution, seemingly needs to be adapted as a function of the disturbances when computing the density, but not for the higher (even) moments. All this has repercussions on those wave problems for which Tsallis hot species densities are needed. For hot species distributions with superthermal wings, only $1 / 3<q<1$ is relevant, even though, at the level of the fluid densities and related variables, the expressions are perfectly continuous at $q=1$ and beyond.

\section{Acknowledgements}

This paper is dedicated to the memory of Padma Kant Shukla, whose untimely death has left us bereft of an excellent and passionate scientist, and of a most loyal friend. Interesting discussions with M. A. Hellberg, I. Kourakis, and G. Williams are gratefully acknowledged.

\section{References}

Cairns, R. A., Mamun, A. A., Bingham, R., Boström, R., Dendy, R. O., Nairn C. M. C. and Shukla, P. K. 1995 Electrostatic 
solitary structures in non-thermal plasmas. Geophys. Res. Lett. 22, 2709-2712.

Hellberg, M.A., Mace, R.L., Baluku, T.K., Kourakis, I. and Saini, N.S. 2009 Comment on mathematical and physical aspects of Kappa velocity distribution [Phys. Plasmas 14, 110702 (2007)]. Phys. Plasmas 16, 094701.

Lima, J.A.S., Silva, R., Jr. and Santos, J. 2000 Plasma oscillations and non-extensive statistics. Phys. Rev. E 61, 3260-3263.

Livadiotis, G. and McComas, D.J. 2009 Beyond kappa distributions: exploiting Tsallis statistical mechanics in space plasmas. J. Geophys. Res. Space Phys. 114, A11105.

Livadiotis, G. and McComas, D. J. 2011 Invariant kappa distribution in space plasmas out of equilibrium. Astrophys. J. 741, 88.
Saberian, E. and Esfandyari-Kalejahi, A. 2013 Langmuir oscillations in a non-extensive electron-positron plasma. Phys. Rev. E 87, 053112.

Tribeche, M., Amour, R. and Shukla, P. K. 2012 Ion acoustic solitary waves in a plasma with non-thermal electrons featuring Tsallis distribution. Phys. Rev. E 85 , 037401.

Tsallis, C. 1988 Possible generalization of Boltzmann-Gibbs statistics. J. Stat. Phys. 52, 479-487.

Vasyliunas, V. M. 1968 A survey of low-energy electrons in the evening sector of the magnetosphere with OGO 1 and OGO 3. J. Geophys. Res. 73, 2839-2884.

Williams, G., Kourakis, I., Verheest, F. and Hellberg, M. A. 2013 Re-examining the Cairns-Tsallis model for ion acoustic solitons. Phys. Rev. E 88, 023103. 